\title{
A Single-Center Review of Prescribing Trends and Outcomes of Corticosteroid Replacement Therapy in Critically III Children with Septic Shock
}

\author{
Scott T. Benken ${ }^{1}$, Tamara K. Hutson ${ }^{2}$, Rhonda L. Gardiner ${ }^{2}$ and Derek S. Wheeler ${ }^{*}, 3,4$ \\ ${ }^{I}$ The James L. Winkle College of Pharmacy, University of Cincinnati, Cincinnati, OH, USA \\ ${ }^{2}$ Division of Pharmacy, Cincinnati Children's Hospital Medical Center, Cincinnati, OH, USA \\ ${ }^{3}$ Division of Critical Care Medicine, Cincinnati Children's Hospital Medical Center, The Kindervelt Laboratory for \\ Critical Care Medicine Research, Cincinnati Children's Research Foundation, Cincinnati, OH, USA \\ ${ }^{4}$ Department of Pediatrics, University of Cincinnati College of Medicine, Cincinnati, OH, USA
}

\begin{abstract}
Recently published consensus treatment guidelines for pediatric sepsis recommend initiating corticosteroid replacement therapy (CRT) for those critically ill children with adrenal insufficiency and refractory shock. The data to support this recommendation is limited, and multiple studies have demonstrated significant variation in both the diagnosis and treatment of adrenal insufficiency and refractory shock in children. In order to better define the variation in practice at our institution, we retrospectively reviewed the experience with CRT in critically ill children with refractory septic shock over a 1-year-period. In addition, as a secondary aim we compared outcomes in critically ill children treated with CRT for variable lengths of time. We found that the initiation of CRT at our center is relatively consistent. However, we noted significant variation in the duration of CRT and whether CRT was gradually tapered or stopped abruptly. The majority of the patients in our cohort received less than the currently recommended duration of 7 days of CRT. There were a higher number of treatment failures in those patients who received CRT for greater than 7 days, suggesting that CRT should be tapered gradually in these patients. There is significant variation in prescribing trends for CRT at our institution, which are likely to be compounded in any multi-center cohort study of CRT in critically ill children with septic shock. Practice variation in CRT should be standardized to address the impact of CRT in this population.
\end{abstract}

Keywords: Sepsis, severe sepsis, adrenal insufficiency, pediatrics.

\section{INTRODUCTION}

Septic shock remains a significant health problem in critically ill children, accounting for close to $\$ 2$ billion per year in healthcare expenditures in the United States alone [1]. Several noteworthy advances have contributed greatly to our understanding of the inherent complexities of the host inflammatory response at the cellular and molecular level in children with septic shock $[2,3]$. However, in spite of these advances, septic shock continues to account for significant morbidity and mortality. Hospital mortality in critically ill children with septic shock approaches $10 \%$, though mortality is slightly higher in children with pre-existing co-morbidities [1]. Comprehensive treatment guidelines for the management of septic shock have been recently published that specifically target the pediatric population [4]. These guidelines emphasize early resuscitation and reversal of shock, early administration of appropriate antibiotic therapy, and maintaining an adequate oxygen delivery using clinically relevant therapeutic endpoints. Importantly, the use of these guidelines has recently been associated with

*Address correspondence to this author at the Division of Critical Care Medicine, Cincinnati Children's Hospital Medical Center, 3333 Burnet Avenue, Cincinnati, OH 45229-3039, USA; Tel: (513) 636-4259; Fax: (513) 636-4267; E-mail: Derek.wheeler@cchmc.org improvement in outcomes $[5,6]$. These management guidelines also emphasize initiating corticosteroid replacement therapy (CRT) in those children with adrenal insufficiency (AI), though there is relatively little data in the pediatric population to support this practice [7].

The potential efficacy of CRT in critically ill adults with septic shock [8] has fueled considerable debate regarding the appropriate definition and management of AI in critically ill children. Unfortunately, the lack of a universally accepted definition has made interpretation of study results complicated and identification of patients who might benefit from CRT difficult. The incidence of AI in critically ill children with septic shock ranges between 9 to $44 \%$, depending upon which particular definition is used [9]. In addition, there is significant variation between available studies in the dose, duration of therapy, concomitant use of mineralocorticoid therapy (i.e. fludrocortisone), and whether a taper is used [10-12]. This lack of consensus precludes any meaningful comparison of studies or the routine practices between different pediatric intensive care units (PICUs). Regardless, there is at least some preliminary evidence that CRT may improve outcome in critically ill children with AI secondary to septic shock $[7,9,13,14]$. We were therefore interested in examining our current practice to determine whether there is significant variation in the diagnosis and treatment of AI in critically ill children with septic shock at 
our institution, as the differences in prescribing trends at a single-institution would likely be magnified further in any multi-center trial enrolling critically ill children with septic shock. In addition, as a secondary aim, we sought to compare outcomes in critically children with septic shock who were treated with CRT with different dosages and for variable lengths of time.

\section{MATERIALS AND METHODS}

\section{Setting}

Cincinnati Children's Hospital Medical Center (CCHMC) is a 523-bed academic, quaternary-care, freestanding children's hospital. It is the only pediatric hospital in the Greater Cincinnati area and serves as a primary referral center for an eight-county area in southwestern Ohio, northern Kentucky, and southeastern Indiana. In fiscal year 2009, CCHMC had over 31,000 admissions and 114,000 emergency department visits and performed nearly 6,000 inpatient surgical procedures and 25,000 outpatient surgical procedures.

\section{Study Participants}

We conducted a retrospective review of all critically ill children admitted to the PICU at our institution with septic shock who were treated with CRT from December 31, 2006 to January 1, 2008. Approval was obtained from our hospital's investigational review board (IRB), and due to the retrospective nature of our study, the need for informed consent was waived. Patients were identified by searching the pharmacy database for all critically ill children with AI secondary to refractory shock who were treated with CRT. Patients were excluded if they had received corticosteroid therapy, for any reason, during the 14 days prior to admission to the PICU, had a history of Addison's disease, or were greater than 18 years of age. In addition, we excluded those children who received CRT following cardiopulmonary bypass for repair or palliation of congenital heart disease $[15,16]$. Septic shock was diagnosed according to the consensus criteria developed by the Society of Critical Care Medicine (SCCM) and the American College of Chest Physicians (ACCP), modified specifically for pediatrics [17]. Refractory shock was defined as the need for vasoactive infusions following $60 \mathrm{~mL} / \mathrm{kg}$ fluid resuscitation.

Our normal practice at Cincinnati Children's Hospital Medical Center is to evaluate all critically ill children with refractory septic shock for possible adrenal insufficiency via the cosyntropin stimulation test, using a computerized physician order entry (CPOE) order set. There are otherwise no formal guidelines or educational programs at our institution for standardizing coynstropin testing or CRT in critically ill children with septic shock. Briefly, a baseline cortisol level is obtained prior to the intravenous administration of cosyntropin at a dose of $0.25 \mathrm{mg}$. The dose of cosyntropin is reduced to $0.015 \mathrm{mg} / \mathrm{kg}$ body weight for critically ill children less than one month of age. Serum cortisol is then measured at 30 and 60 minutes following administration of cosyntropin. Absolute adrenal insufficiency (AAI) is defined as a baseline cortisol level < $10 \mu \mathrm{g} / \mathrm{dL} \quad(<276 \mathrm{nmol} / \mathrm{L})$, while relative adrenal insufficiency (RAI) is defined as incremental change at 60 minutes $(\Delta 60)$ of $<9 \mu \mathrm{g} / \mathrm{dL}(<248 \mathrm{nmol} / \mathrm{L})$ [7]. In general,
CRT is administered to those critically ill children meeting criteria for either AAI or RAI. The dose of hydrocortisone, duration of therapy, and the length of the hydrocortisone taper (if used) is left to the discretion of the attending physician, based upon the results of the cosyntropin stimulation test and the patient's clinical condition or status. CRT is initiated, when indicated, as soon as the results of the cosyntropin stimulation test are made available.

\section{Study Design and Data Collection}

Patient demographic information including age, weight, height, Pediatric Risk of Mortality (PRISM)-III score [18], and diagnosis were collected via review of the electronic medical record (EMR), pharmacy database, and PICU database. The inotrope score [19] was calculated at one hour prior to initiation of CRT, 24 hours after initiation of CRT, and at subsequent 24 hour intervals during CRT until vasopressor therapy was discontinued or the patient expired. Briefly, the inotrope score is calculated as the sum of all inotrope doses, correcting for potency (dopamine, dobutamine $=1$ point for every $1 \mu \mathrm{g} / \mathrm{kg} / \mathrm{min}$; milrinone $=10$ points for every $1 \mu \mathrm{g} / \mathrm{kg} / \mathrm{min}$, and epinephrine $=100$ points for every $1 \mu \mathrm{g} / \mathrm{kg} / \mathrm{min}$ ) [19]. The duration of inotropic support was also determined. The results of the cosyntropin stimulation test, including the dose of cosyntropin administered and serum cortisol measured at baseline, 30 minutes, and 60 minutes after cosyntropin was recorded. The daily hydrocortisone dose per BSA, the total duration of CRT, the duration of CRT before beginning a taper (where applicable), and the duration of the corticosteroid taper was abstracted from the pharmacy database and EMR. Total length of stay in the PICU, survival to PICU discharge, and 28-day survival were determined.

\section{Data Analysis}

Abstracted data was tabulated in a Microsoft Excel 2003 spreadsheet (Microsoft, Redmond, WA) and analyzed using Sigmastat for Windows, version 3.11 (Systat Software, Inc, San Jose, CA). The indication for CRT was stratified into one of four groups, based upon a low baseline cortisol $(\leq 10$ $\mu \mathrm{g} / \mathrm{dL}, 276 \mathrm{nmol} / \mathrm{L})$, low incremental change following cosyntropin $(\Delta 60 \leq 9 \mu \mathrm{g} / \mathrm{dL}, 248 \mathrm{nmol} / \mathrm{L})$, both, or neither (i.e. empiric therapy). We calculated the total duration of CRT (prospectively defined as the time from initiation to completion of CRT), the duration of CRT "stress dosing" (prospectively defined as the time from initiation of CRT to the initiation of a taper, when applicable), and the duration of the taper/wean (prospectively defined as the time when the dose of corticosteroid was gradually decreased until the time CRT was discontinued). Continuous variables were expressed as median (interquartile range) due to the nonparametric nature of the data. In order to determine whether the level of inotropic support impacted the decision to initiate CRT, we stratified patients into tertiles based upon the initial inotrope score and compared the total duration of CRT, duration of CRT "stress dosing," and duration of CRT taper (when applicable) between groups using one-way ANOVA. We also compared 28-day mortality between groups via Fisher's exact test.

Previous adult studies and a consensus guideline on the management of adrenal insufficiency in critically ill adults have recommended at least a minimum of 7 days of therapy, 
once CRT is initiated [20]. We further stratified patients into two groups based upon the total duration of CRT ( $>7$ days CRT $v s \leq 7$ days CRT). We prospectively defined "weaning failure" as a greater than $50 \%$ increase in the inotrope score after discontinuing CRT or if CRT was re-initiated for any reason after beginning a taper. We compared 28-day mortality between each group via Chi square test. We used Bonferroni's correction for multiple comparisons, and a pvalue $<0.05$ was considered statistically significant.

\section{RESULTS}

During the 12-month period of review, 51 critically ill children were treated with CRT (Table 1). Consistent with the previously described effects of etomidate on the hypothalamic-pituitary-adrenal (HPA) axis, approximately $20 \%$ of our patients had received treatment with etomidate at some point during the 48 hours before initiation of CRT [21]. Adrenal insufficiency was diagnosed based upon a cosyntropin stimulation test performed within 24 hours of admission to the PICU. All 51 children in our cohort had evidence of refractory septic shock based upon a median inotrope score at baseline of 13 (IQR 9, 39). CRT was initiated in 26/51 (51\%) children due to a low baseline cortisol (AAI), while CRT was initiated in 19/51 (37\%) due to a low $\Delta 60$ (RAI). CRT was initiated in $5 / 51$ children $(10 \%)$ due to both a low baseline cortisol and a low $\Delta 60$. CRT was initiated empirically in one child in the absence of either a low baseline cortisol or low $\Delta 60$. The majority of critically ill children $(n=46 / 51,90 \%)$ were treated with hydrocortisone at a total dose of $50 \mathrm{mg} / \mathrm{m}^{2} \mathrm{BSA} /$ day divided every 6 hours. Three children $(6 \%)$ were treated with $>50 \mathrm{mg} / \mathrm{m}^{2} \mathrm{BSA} /$ day and two children $(4 \%)$ were treated with $<50 \mathrm{mg} / \mathrm{m}^{2} \mathrm{BSA} /$ day for reasons that were not specified in the medical record. None of the children were treated with concomitant mineralocorticoid replacement therapy (e.g., fludrocortisones). A taper was used in $31 / 51$ (60.7\%) children with a median duration of 2 days (IQR 0, 7.5). CRT was discontinued abruptly in the remaining children. The median duration of CRT prior to initiating the taper in the cohort was 5 days (IQR 3, 8.5). The overall mortality in our cohort was $17.6 \%$, which is comparable to the reported mortality in previously published series of adrenal insufficiency secondary to pediatric septic shock $[9,14,21$ 25].

Table 1. Patient Demographics $(n=51)$

\begin{tabular}{|l|r|}
\hline Age (mean), yrs & $5.1(0.1-17)$ \\
\hline Weight, kg (mean \pm SEM) & $19.0 \pm 2.2$ \\
\hline Gender (M:F) & $25: 26$ \\
\hline PRISM-III score, median (IQR) & $12.8(1-48)$ \\
\hline Baseline Inotrope score, median (IQR) & $30.7(3-300)$ \\
\hline Etomidate 48 hours prior to starting hydrocortisone, n (\%) & $10(19.6 \%)$ \\
\hline
\end{tabular}

In order to determine whether the severity of illness at the initiation of CRT influenced prescribing trends, we stratified patients into tertiles based upon the initial inotrope score (Fig. 1). There were no significant differences between the three groups with respect to the total duration of CRT, the duration of "stress dosing" of CRT (defined as the duration on stress-dose hydrocortisone of at least 50 $\mathrm{mg} / \mathrm{m}^{2} /$ day), and the duration of the taper (when applicable). There were no significant differences in 28-day mortality in the highest inotrope based upon initial inotrope score tertile $\left(1^{\text {st }}\right.$ tertile: $11.8 \% ; 2^{\text {nd }}$ tertile: $11.8 \% ; 3^{\text {rd }}$ tertile: $27.8 \%$; $\mathrm{p}=0.3)$.

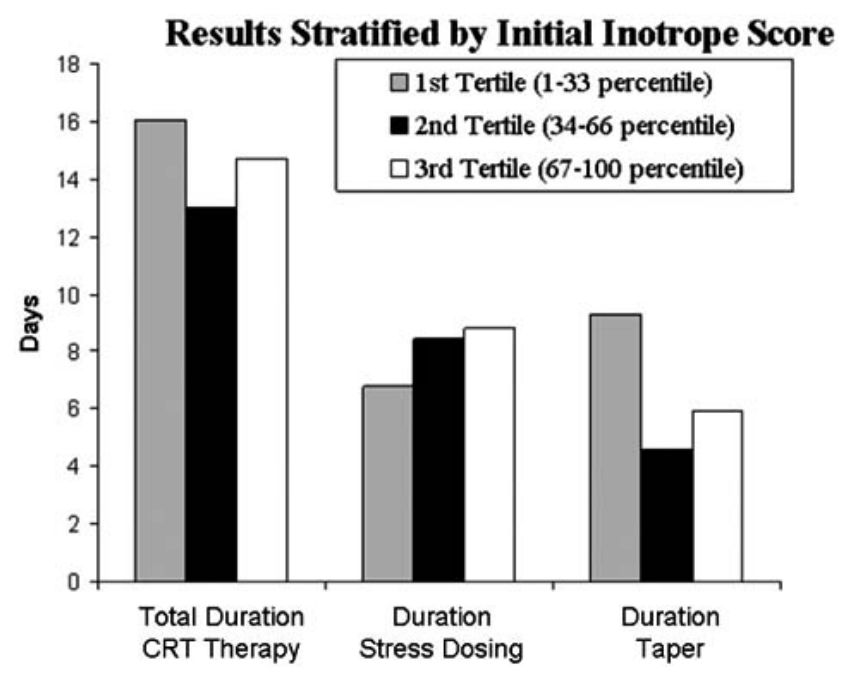

Fig. (1). CRT stratified by initial inotrope score.

Previous adult studies and a consensus guideline on the management of adrenal insufficiency in critically ill adults have recommended at least a minimum of 7 days of therapy, once CRT is initiated [20]. We therefore stratified patients by the duration of "stress dosing" CRT into two groups ( $>7$ days "stress dosing" CRT $v s \leq 7$ days "stress dosing" CRT). Thirty-six (71\%) patients were treated with "stress dosing" CRT for $\leq 7$ days, while the remainder $(15 / 51,29 \%)$ received "stress dosing" CRT for $>7$ days (median duration 13 days, IQR 9, 18). While there were no significant differences between these two groups, with respect to demographics, severity of illness, or baseline inotrope score, the number of weaning failures was significantly greater in patients that were treated for longer than 7 days CRT (Table 2). The number of patients that failed the taper in each group was four and nine $(11.1 \%$ and $60 \%)$ respectively $(\mathrm{p}<0.001)$. Regardless, there was no difference in mortality between these two groups of patients.

\section{DISCUSSION}

We retrospectively reviewed the prescribing practices for CRT in critically ill children with septic shock at our institution over a 1-year period and noted significant variation with regards to duration of CRT and whether CRT was gradually tapered or stopped abruptly. Our data suggested that the initiation of CRT at our center is relatively consistent, with only one patient receiving CRT in the absence of a cosyntropin stimulation test. The majority of the patients in our cohort received less than the recommended seven days of CRT, though the period of study occurred prior to the release of the consensus guidelines [20]. There were a higher number of weaning failures in those patients who received CRT for greater than 7 days, suggesting that CRT should be tapered gradually in these patients.

We did not specifically analyze the factors that were associated with variation in prescribing practices for CRT. 
Table 2. Patient Demographics and Outcome Based Upon Duration of CRT

\begin{tabular}{|l|c|c|}
\hline & $\leq 7$ Days Stress Dose CRT & $>$ 7 Days Stress CRT \\
\hline \hline N (\%) & $36(70.6 \%)$ & $15(29.4 \%)$ \\
\hline Duration of Inotropes (h), median (IQR) & $76(41,83)$ & $59(56.75,217.75)$ \\
\hline Duration of Hydrocortisone Taper (days), median (IQR) & $2(0,6)$ & $5(0.75,13.5)$ \\
\hline Weaning failure, $\mathbf{n}(\%)$ & $4 / 36(11.1 \%)^{\dagger}$ & $9 / 15(60 \%)^{\dagger}$ \\
\hline Mortality (\%) & $6 / 36(16.7 \%)^{\ddagger}$ & $3 / 15(20 \%)^{\ddagger}$ \\
\hline
\end{tabular}

All of the treatment decisions regarding CRT were made by the attending PICU physician. All of the attending PICU physicians at our institution have completed approved pediatric critical care medicine fellowship training programs and are either board-eligible or board-certified in pediatric critical care medicine. The experience ranges between 2 to 15 years of practice following completion of training. It is possible that varying levels of experience or differences in fellowship training contributed to the variation in practice. Alternatively, patient factors may have biased treatment decisions. However, neither the severity of illness (based upon initial inotrope score) nor the time on vasoactive infusions affected the duration of CRT or use of a taper. Regardless, we feel that a multi-center study will be necessary to further define the variation in prescribing patterns for CRT in critically ill children.

Corticosteroids have been used in the management of critically ill patients with septic shock for the last several decades [26]. The approach until the early 1980's focused on administering very high, supraphysiologic doses of corticosteroids in an attempt to block the host inflammatory response - consistent with the prevailing theory of the pathophysiology of sepsis at the time. Unfortunately, large, multi-center, randomized, placebo-controlled trials failed to show any benefits to this practice $[27,28]$. Two subsequent meta-analyses $[29,30]$ failed to demonstrate any benefit to high-dose corticosteroid administration in this patient population, and the practice was largely abandoned [7]. More recently, there has been a resurgence of interest in the use of corticosteroids in the management of critically ill patients with septic shock. Following the promising results of smaller studies that suggested a benefit to moderate-dose corticosteroids [31, 32], Annane and co-workers [33] conducted a multi-center, randomized, placebo-controlled, double-blind trial comparing the use of stress-dose hydrocortisone $(50 \mathrm{mg}$ i.v. every 6 hours $)$ and fludrocortisone (50 $\mu \mathrm{g}$ once daily) or placebo in critically ill adults with septic shock and adrenal insufficiency (as determined by an inadequate response to cosyntropin stimulation test). While the trial suffered from some methodologic concerns [26], there was a significant reduction in the duration of vasopressor therapy and 28-day mortality in patients with adrenal insufficiency who were randomized to the treatment group.

The results of the Corticosteroid Therapy of Septic Shock (CORTICUS) trial [34] were recently published, in which hydrocortisone treatment shortened the duration of time to shock reversal in patients with an inadequate cortisol response to cosyntropin, as well as those patients who did respond with an adequate cortisol response to cosyntropin. However, there was no difference in 28-day mortality in either the responder group or non-responder group in patients randomized to hydrocortisone treatment $v s$ placebo. The trial was underpowered to detect a difference in mortality, as the trial was prematurely terminated after only 500 of the planned 800 subjects were enrolled due to slow enrollment.

Unfortunately, there have been no prospective, randomized, placebo-controlled trials of CRT in critically ill children with septic shock. Despite the lack of available data, CRT is commonly prescribed in the vast majority of PICUs throughout North America $[10,11]$ and the United Kingdom [13]. For example, in one recent survey, $51 \%$ of Canadian pediatric intensivists stated that they would treat refractory septic shock with CRT [10]. In an electronic survey of pediatric intensivists who subscribe to the PICUList e-mail discussion group, $48 \%$ of those responding stated that they routinely prescribe CRT based upon the presence of refractory septic shock and in the absence of cosyntropin testing. Moreover, $68 \%$ of those surveyed stated that they would not participate in any study that would potentially randomize critically ill children with refractory septic shock to a placebo-arm [11]. All three surveys noted wide variation in both prescribing practices and diagnosis of adrenal insufficiency $[10,11,13]$, consistent with the results of the current study.

The current consensus guideline on the management of adrenal insufficiency in critically ill adults recommends at least a minimum of 7 days of therapy, once CRT is initiated [20]. Given the relative paucity of data in critically ill children, we stratified patients based upon the duration of CRT ( $>7$ days CRT vs $\leq 7$ days CRT). While there was no difference in mortality between these two groups of patients, the number of weaning failures was significantly greater in patients that were treated for longer than 7 days CRT, suggesting that a taper is warranted in this population rather than abruptly terminating therapy. The optimal duration of therapy in both critically ill children and adults with AI is not known. However, the consensus guidelines currently recommend tapering CRT rather than stopping treatment abruptly.

Our findings are certainly limited by the retrospective nature of our study and may not be applicable outside our own institution. However, the significant variation in CRT 
prescribing practices at our single-institution are likely to be compounded even further in any multi-center, cohort study, making it difficult, if not impossible, to compare and analyze outcomes in critically ill children receiving CRT for refractory septic shock. The significant variation in CRT practice noted by the aforementioned surveys $[10,11,13]$ would appear to support this conclusion.

Given the paucity of clear-cut evidence for CRT in both critically ill children [7] and adults [34], we feel that a prospective, randomized, placebo-controlled trial of CRT in the PICU is clearly warranted $[11,35]$. However, there are several potential barriers to the completion of such a trial [7]. First and foremost, as the results of the current study suggest, there is significant variation in practice regarding both the diagnosis and treatment of AI in critically ill children with septic shock. Moreover, there is significant variation in practice with regards to the diagnosis and management of critically ill children with septic shock. Second, critically ill children with septic shock have a relatively low mortality rate compared to adults, such that thousands of patients in both the therapy and control groups would be necessary for a sufficiently powered study using the traditional 28-day mortality as primary outcome. Finally, there is perhaps lack of equipoise for conducting a trial in which critically ill children are randomized to placebo or CRT. With these substantial barriers in mind, we believe that a multi-center cohort study using historical controls and in which practice variation is minimized could provide important, supportive evidence for CRT in this population. We suggest that CRT prescribing practices should be standardized in order to minimize variation and allow for meaningful comparison between centers.

\section{ACKNOWLEDGEMENT}

Supported by the National Institutes of Health, 5KO8GM077432 and 1R03HD058246.

\section{REFERENCES}

[1] Watson RS, Carcillo JA, Linde-Zwirble WT, Clermont G, Lidicker $\mathrm{J}$, Angus DC. The epidemiology of severe sepsis in children in the United States. Am J Respir Crit Care Med 2003;167:695-701.

[2] Cornell TT, Wynn J, Shanley TP, Wheeler DS, Wong HR. Mechanisms and regulation of the gene-expression response to sepsis. Pediatrics 2010; 125: 1248-58.

[3] Wynn J, Cornell TT, Wong HR, Shanley TP, Wheeler DS. The host response to sepsis and developmental impact. Pediatrics 2010; 125: 1031-41.

[4] Brierley J, Carcillo JA, Choong K, et al. Clinical practice parameters for hemodynamic support of pediatric and neonatal septic shock: 2007 update from the American College of Critical Care Medicine. Crit Care Med 2009; 37: 666-88.

[5] de Oliveira CF, de Oliveira DS, Gottschald AF, et al. ACCM/PALS haemodynamic support guidelines for paediatric septic shock: An outcomes comparison with and without monitoring of central venous oxygen saturation. Intensive Care Med 2008; 34(6): 1065-75.

[6] Carcillo JA, Kuch BA, Han YY, et al. Mortality and morbidity after use of PALS/APLS by community physicians. Pediatrics 2009; 124: 500-8.

[7] Zimmerman JJ. A history of adjunctive glucocorticoid treatment for pediatric sepsis: Moving beyond steroid pulp fiction toward evidence-based medicine. Pediatr Crit Care Med 2007; 8: 530-9.

[8] Annane D, Sebille V, Charpentier C, et al. Effect of treatment with low doses of hydrocortisone and fludrocortisone on mortality in patients with septic shock. JAMA 2002; 288: 862-71.
[9] Pizzaro CF, Troster EJ, Damiani D, Carcillo JA. Absolute and relative adrenal insufficiency in children with septic shock. Crit Care Med 2005; 33: 855-9.

[10] Menon K, Lawson M. Identification of adrenal insufficiency in pediatric critical illness. Pediatr Crit Care Med 2007; 8: 276-8.

[11] Zimmerman JJ. Testing the waters. Pediatr Crit Care Med 2007; 8: 305-7.

[12] Annane D. Time for a consensus definition of corticosteroid insufficiency in critically ill patients. Crit Care Med 2003; 31: 1868-9.

[13] Hildebrandt T, Mansour M, Al Samsam R. The use of steroids in children with septicemia: Review of the literature and assessment of current practice in PICUs in the UK. Pediatr Anesthesia 2005; 15: 358-65.

[14] Markovitz BP, Goodman DM, Watson RS, Bertoch D, Zimmerman J. A retrospective cohort study of prognostic factors associated with outcome in pediatric severe sepsis: What is the role of steroids? Pediatr Crit Care Med 2005; 6: 270-4.

[15] Shore S, Nelson DP, Pearl JM, et al. Usefulness of corticosteroid therapy in decreasing epinephrine requirements in critically ill infants with congenital heart disease. Am J Cardiol 2001; 88: 5914.

[16] Suominen PK, Dickerson HA, Moffett BS, et al. Hemodynamic effects of rescue protocol hydrocortisone in neonates with low cardiac output syndrome after cardiac surgery. Pediatr Crit Care Med 2005; 6: 655-9.

[17] Goldstein B, Giroir B, Randolph A. International pediatric sepsis consensus conference: definitions for sepsis and organ dysfunction in pediatrics. Pediatr Crit Care Med 2005; 6: 2-8.

[18] Pollack MM, Patel KM, Ruttimann UE. PRISM III: an updated Pediatric Risk of Mortality score. Crit Care Med 1996; 24: 743-52.

[19] Domico M, Liao P, Anas N, Mink RB. Elevation of brain natriuretic peptide levels in children with septic shock. Pediatr Crit Care Med 2008; 9: 478-83.

[20] Marik PE, Pastores SM, Annane D, et al. Recommendations for the diagnosis and management of corticosteroid insufficiency in critically ill adult patients: Consensus statements from an international task force by the American College of Critical Care Medicine. Crit Care Med 2008; 36: 1937-49.

[21] den Brinker M, Joosten KF, Liem O, et al. Adrenal insufficiency in meningococcal sepsis: Bioavailable cortisol levels and impact of interleukin-6 levels and intubation with etomidate on adrenal function and mortality. J Clin Endocrinol Metab 2005; 90: 5110-7.

[22] Sarthi M, Lodha R, Vivekanandhan S, Arora NK. Adrenal status in children with septic shock using low-dose stimulation test. Pediatr Crit Care Med 2007; 8: 23-8.

[23] Hatherill M, Tibby SM, Hilliard T, Turner C, Murdoch IA. Adrenal insufficiency in septic shock. Arch Dis Child 1999; 80: 51-5.

[24] Menon K, Clarson C. Adrenal function in pediatric critical illness. Pediatr Crit Care Med 2002; 3: 112-6.

[25] Casartelli CH, Garcia PC, Branco RG, Einloft PR, Tasker RC. Adrenal response in children with septic shock. Intensive Care Med 2007; 33: 1609-13.

[26] Mesotten D, Vanhorebeek I, Van den Berghe G. The altered adrenal axis and treatment with glucocorticoids during critical illness. Nat Clin Pract Endocrinol Metab 2008; 4: 496-505.

[27] Group. VASSCS. Effect of high-dose glucocorticoid therapy on mortality in patients with clinical signs of systemic sepsis. N Engl J Med 1987; 317: 659-65.

[28] Bone RC, Fisher CJ, Jr., Clemmer TP, Slotman GJ, Metz CA, Balk RA. A controlled clinical trial of high-dose methylprednisolone in the treatment of severe sepsis and septic shock. N Engl J Med 1987; 317: 653-8.

[29] Lefering R, Neugebauer EA. Steroid controversy in sepsis and septic shock: A meta-analysis. Crit Care Med 1995; 23: 1294-303.

[30] Cronin L, Cook DJ, Carlet J, et al. Corticosteroid treatment for sepsis: A critical appraisal and meta-analysis of the literature. Crit Care Med 1995; 23: 1430-9.

[31] Bollaert PE, Charpentier C, Levy B, Debouverie M, Audibert G, Larcan A. Reversal of late septic shock with supraphysiologic doses of hydrocortisone. Crit Care Med 1998; 26: 645-50.

[32] Briegel J, Forst H, Haller M, et al. Stress dose of hydrocortisone reverse hyperdynamic septic shock: A prospective, randomized, double-blind, single-center study. Crit Care Med 1999; 27: 723-32. 
[33] Annane D, Sebille V, Charpentier C, et al. Effect of treatment with low doses of hydrocortisone and fludrocortisone on mortality in patients with septic shock. JAMA 2002; 288: 862-71.
[34] Sprung CL, Annane D, Keh D, et al. Hydrocortisone therapy for patients with septic shock. N Engl J Med 2008; 358: 111-24.

[35] Annane D, Bellissant E, Bollaert PE, et al. Corticosteroids in the treatment of severe sepsis and septic shock in adults: A systematic review. JAMA 2009; 301: 2362-75.

Received: June 18, 2010

Revised: July 26, 2010

Accepted: August 6, 2010

(C) Benken et al.; Licensee Bentham Open.

This is an open access article licensed under the terms of the Creative Commons Attribution Non-Commercial License (http://creativecommons.org/licenses/ by-nc/3.0/) which permits unrestricted, non-commercial use, distribution and reproduction in any medium, provided the work is properly cited. 